\title{
Novel Insights into the Regulation of the Timeless Protein
}

\author{
Lesley J. Ashmore, Sriram Sathyanarayanan, David W. Silvestre, Mark M. Emerson, Peter Schotland, and Amita Sehgal \\ Howard Hughes Medical Institute, Department of Neuroscience, University of Pennsylvania Medical School, Philadelphia, Pennsylvania 19104
}

In the Drosophila circadian clock, period ( per) and its partner, timeless (tim), play a central role in the negative limb of an autoregulatory feedback loop. Unlike per, the dosage of which affects the frequency (tau) of the circadian cycle, we found that increasing copies of the tim gene has no effect on clock period length. The use of the tim promoter to express per results in a shortening of circadian period, also indicating that the regulation of tim is different from that of per. Drosophila TIM is similar to the mammalian circadian protein mPER2 in that it shuttles independently between the nucleus and cytoplasm both in vivo and in vitro. Contrary to the current model that PER and TIM heterodimerization is a prerequisite for their nuclear entry, PER is not required to transport TIM into nuclei, although it influences TIM localization and vice versa. Blocking nuclear export led to increased nuclear expression of TIM in S2 cells and in wild-type and per ${ }^{01}$ larvae, suggesting that PER may be required for nuclear retention of TIM. Unlike PER, nuclear TIM alone has no ability to repress transcription. We propose that TIM drives cycles of PER expression by regulating its stability, and in turn, PER retains TIM in the nucleus, either for the regulation of its own stability or for a novel nuclear role of TIM.

Key words: period; timeless; rhythms; cycle; biological clocks; transcription factors; nuclear export; LMB

\section{Introduction}

Nearly all organisms have an endogenous $24 \mathrm{hr}$ circadian clock. This clock is normally entrained to the organism's environment by light or other environmental cues and controls daily hormonal, behavioral, and social rhythms. In Drosophila melanogaster, the clock that controls rest-activity rhythms is located in the brain, predominantly in the small ventrolateral neurons $\left(\mathrm{LN}_{\mathrm{v}} \mathrm{s}\right)$. Within these $\mathrm{LN}_{\mathrm{v}} \mathrm{s}$, period (per) and timeless (tim) transcript and protein levels oscillate with a daily rhythm (for review, see Williams and Sehgal, 2001; Stanewsky, 2003). PER and TIM form a heterodimer (PER-TIM) that enters the nucleus midway through the night and negatively regulates per and tim transcription by blocking the activity of transcriptional activators Clock $(d C l k)$ and $c y c l e(c y c)$. The inhibition is released in the morning when the levels of TIM and PER decrease.

Between these two proteins, the main focus of the field has been on PER regulation and function. Early on, Smith and Konopka (1982) found that changing the gene dosage of per altered the period of the clock in an inverse manner; decreasing per copy number lengthened the period of the clock, and vice versa. This led to the hypothesis that PER is a state variable of this system, a timekeeper molecule with levels that indicate to the organism what time of day it is. The partner of PER, TIM, is important both for PER function [PER is unstable when not bound to TIM and requires TIM for nuclear expression (Vosshall

Received April 29, 2003; revised June 27, 2003; accepted July 1, 2003.

This work was supported by grants from the National Institutes of Health (NIH) and National Science Foundation to A.S. A.S. is an Associate Investigator of the Howard Hughes Medical Institute. L.A. was supported by the Behavioral Neuroscience training grant from the NIH. We thank Steve Kay for providing the pAct-dClock, pAct-per, and perEhsp-luc constructs to SS, and members of the Sehgal lab for useful discussion.

Correspondence should be addressed to Amita Sehgal, Department of Neuroscience, 232 Stemmler Hall, Thirtyfifth and Hamilton Walk, Philadelphia, PA 19104. E-mail: amita@mail.med.upenn.edu.

M. M. Emerson's present address: Department of Neurobiology, Harvard University, Boston, MA 02115. Copyright @ 2003 Society for Neuroscience $\quad$ 0270-6474/03/237810-10\$15.00/0 et al., 1994; Price et al., 1995; Saez and Young, 1996)] and as a point of photic input to the clock. TIM is actively degraded at dawn through light-independent as well as light-triggered mechanisms (Hunter-Ensor et al., 1996; Lee et al., 1996; Myers et al., 1996; Zeng et al., 1996; Naidoo et al., 1999). However, other than its effects on PER, the role of TIM in the actual clock mechanism has not been addressed. Is TIM a state variable of the clock? Can TIM repress transcription in the absence of PER? These are the questions we initially sought to answer in this study.

We examined the role of TIM in the circadian clock in two ways. First we addressed whether changes in TIM levels and TIMPER levels, or both, affect the length of the circadian period and found that the clock is not sensitive to tim dosage. Second, to determine the role of TIM in feedback regulation, we assayed the ability of nuclear TIM to affect activity of dCLK and CYC in the absence of PER. Without PER, TIM showed no ability to repress transcription. We also discovered that TIM, like the mammalian clock protein mPER2 (Yagita et al., 2002), is regulated via active CRM1/Exportin 1-dependent nuclear export mechanisms. PER does not appear to be regulated by this pathway. Dimerization with PER is not a prerequisite for nuclear entry; however, PER is necessary for nuclear retention of TIM. Although PER is also affected by TIM localization, PER is probably the main target of mechanisms that control the timing of nuclear entry.

\section{Materials and Methods}

Generation of transgenes. Tim 4 and Tim 7 constructs were described in Ousley et al. (1998). Tim 4 contains $4.3 \mathrm{~kb}$ of the upstream sequence of the tim gene fused to a tim cDNA. Tim 7 contains the same promoter region as Tim 4, but most of the coding portion has been replaced with genomic sequence. To generate Per 1, we first cloned an EcoRI fragment containing full-length per cDNA from pEMBL into BC1. BC1 was constructed by cloning the EcoRI-PstI fragment (563 bp) of pCaSpeR-hs (Thummel and Pirrotta, 1992) into pBluescript. This allowed us to excise per from BC1 with StuI (originally from pCaSpeR-hs) and KpnI (origi- 
nally from pBluescript) and clone it into the unique sites SwaI and KpnI behind the tim promoter in pCaSpeR4. Thus, Per 1 contains the identical tim upstream promoter region as Tim 4 and Tim 7, fused to per cDNA (see Fig. 2).

To allow nuclear expression of TIM in the absence of PER, two different constructs were generated using a tim cDNA capable of rescuing behavioral rhythms subcloned into the hsp70 promoter-driven expression vector pCaSpeR-hs, described in Naidoo et al. (1999). In the first modified construct, we added a nuclear localization signal (NLS) to the C-terminal end of full-length tim. A PCR fragment containing the sequence for the SV40 Large T-antigen NLS (PKKKRKV) was amplified from a tim cDNA using the following primers: $3^{\prime}$-(5'-ATGCGATATCTCATACCTTACGCTTCTTCTTAGGGTGATAGTGGGGCACCCGGATCTCGGTTCGCTCAAGTC-3') and $5^{\prime}=\left(5^{\prime}\right.$-GGCCGTGGAGAAGGCACACG-3'). The $3^{\prime}$ primer contained the NLS and an EcoRV restriction site. The resulting fragment was cloned into the EagI and EcoRV restriction sites of tim and named hstim $^{+N L S}$. The second nuclear tim construct, hs-tim $\Delta$ CLD, had the Cterminal 150 amino acids, identified as the cytoplasmic localization domain (CLD) (Saez and Young, 1996) removed. The tim EagI-EcoRV fragment was replaced with a PCR product that lacked the CLD (codon 1228 was replaced by a stop codon). The primers $5^{\prime}=\left(5^{\prime}\right.$-GGCCGTGGAGAAGGCACACG$\left.3^{\prime}\right)$ and $3^{\prime}=\left(5^{\prime}\right.$-CTATAGTCGACGAGGAGCCAACGCAA-3' $)$ were used to generate this PCR fragment. These two modified constructs were then cloned into the multiple cloning site of a $\mathrm{P}$ (pCasPer-hs) vector and injected into $y w, K i \Delta 2-3$ flies for germline transformation.

pAct- $d C l o c k$, pAct- $d$ per, and perE-hsp-luc were provided by Steve Kay and the ie $\beta$-galactosidase $(\beta$-gal) reporter was provided by K. P. Gopinathan.

Preparation of transgenic strains. Tim 4, Tim 7, and Per 1 constructs were inserted into the Drosophila genome through P element-mediated transformation. Yw/yw; Ki $\Delta 2-3$ eggs were injected with the constructs. Multiple independent insertion lines containing each transgene were created from surviving injected eggs. The Tim 4-7 and Per 1-A and 1-B lines were crossed to create fly lines containing multiple copies of per and tim transgenes in wild-type backgrounds.

Locomotor assays. Flies between 1 and $14 \mathrm{~d}$ old were isolated from stocks and entrained in a $12 \mathrm{hr}$ light/dark (L/D) incubator at $25^{\circ} \mathrm{C}$ for at least $3 \mathrm{~d}$. Each fly was then inserted into individual locomotor tubes supplemented with $5 \%$ sucrose, $1 \%$ agar. The flies were transferred to dark/dark, and their locomotor activity was monitored using the Trikinetics system. Actogram and periodogram analyses of Tim 4, Tim 7, Per 1 , and Per $1 \times$ Tim 4 locomotor data were performed using the Tau program.

Antibodies and Western analysis. Antibodies used in this study were UPR 8 (Hunter-Ensor et al., 1996), UPR 34, and TIM 991. UPR 34 was raised in rats using full-length PER expressed in the baculovirus system as an immunogen. Rabbit anti-pigment dispensing factor (PDF) antibodies were raised using synthesized amidated PDF peptide.

Adult flies were isolated from stocks and placed in 5\% sucrose, $1 \%$ agar bottles for collection. They were then entrained for at least three full cycles in a $12 \mathrm{hr} \mathrm{L} / \mathrm{D}$ incubator at $25^{\circ} \mathrm{C}$. Flies were then collected at either Zeitgeber (ZT) 16.5 (PER Westerns) or ZT 21 (TIM Westerns) on dry ice. Protein extracts were prepared from isolated fly heads by homogenization using a small handheld homogenizer (IKA Labortechnik) on ice in homogenization buffer $(150 \mathrm{~mm} \mathrm{NaCl}, 1 \%$ Triton X-100, $50 \mathrm{~mm}$ Tris$\mathrm{HCl}$, pH 7.6, 10 mm EDTA, $1 \times$ Roche Complete protease inhibitor mixture, prepared according to manufacturer's instructions). Protein samples $(80 \mu \mathrm{g})$ were loaded onto a $6 \%$ SDS-polyacrylamide gel, separated, and transferred overnight to a nitrocellulose blot. The blots were blocked either for $1 \mathrm{hr}$ at $25^{\circ} \mathrm{C}$ or overnight at $4^{\circ} \mathrm{C}$ using blocking solution $(8 \%$ milk, $2 \%$ BSA in PBS). Primary antibodies were diluted in the same blocking solution at 1:1000 for UPR 8 and 1:10,000 for UPR 34. Blots were then incubated with secondary antibodies (HRP-conjugated antirat) at a dilution of 1:1000, and PER/TIM was visualized with an ECL detection kit (Amersham Biosciences, Arlington Heights, IL). After stripping, blots were restained with commercial monoclonal antibodies to HSP70 (Sigma, St. Louis, MO) for use as a loading control in PER blots.

S2 cell transfections and immunofluorescence. For staining, Drosophila S2 cells were seeded onto six-well plates at $\sim 1 \times 10^{6}$ cells per well. After
6-12 hr, 500 ng of hs-tim or pAct-per DNA, or both, was transfected into the cells via Cellfectin-assisted transformation (Invitrogen, San Diego, CA). Forty-eight hours after transfection, cells were heat shocked at $37^{\circ}$ for $30 \mathrm{~min}$ to induce TIM expression. In some experiments the cells were heat shocked with leptomycin B (LMB) (or vehicle) added to the medium (at a final concentration of $1-10 \mathrm{ng} / \mathrm{ml}$, as indicated in Figure legends). Two hours after induction, cells were gently rinsed with $1 \times$ PBS, spun at $1000 \times g$ for $1 \mathrm{~min}$, resuspended in 100-200 $\mu \mathrm{l}$ of $1 \times$ PBS, and spread on $22 \times 50 \mathrm{~mm}$ coverslips $(\sim 100 \mu \mathrm{l}$ each slip). After $10 \mathrm{~min}$, the PBS was gently removed, and attached cells were fixed in fresh $4 \%$ paraformaldehyde in PBS. Cells were rinsed with PBS and then blocked for $1 \mathrm{hr}$ in blocking solution ( $10 \%$ normal donkey serum, $0.1 \%$ Triton X-100 in PBS), incubated in primary antibody (1:10,000 in block), and rinsed three times in PBS (10 min each). Cells were then incubated in secondary antibody (FITC-conjugated donkey anti-rat and/or cyanine $3\left(\mathrm{CY}^{*}\right)$ conjugated donkey anti-rabbit, each diluted 1:10,000 in block) for $1 \mathrm{hr}$, rinsed three times $(10 \mathrm{~min})$, and mounted in $4^{\prime}-6^{\prime}$ diamidino-2phenylindole (DAPI)-containing medium (Vector Laboratories, Burlingame, CA).

Each sample slide was scored blind ( 50 cells per slide), using DAPI to determine the approximate location of the nucleus. Cells were divided into categories: cells in which most of the TIM/PER signal was nuclear, cells in which most of the signal was cytoplasmic, and cells in which the signal was distributed evenly (uniform). Some slides were scored independently by a second experimenter, and the results were compared for consistency.

In vitro transcription assays. The effects of various TIM deletions on dCLK-CYC-mediated transcription in S2 cells were tested as described in Darlington et al. (1998) with a few modifications. A luciferase reporter plasmid, perE-hsp-luc (E-box luc), containing four tandem repeats of per E-box and a minimal $h s p$ promoter (10 ng) was transfected into S2 cells using Cellfectin (Invitrogen). Cotransfection of pAct- $d$ Clock (1 ng) activated transcription from the E-box reporter construct. The effect of PER and TIM on dCLK-CYC-mediated transcription was tested by cotransfecting pAct-dper and hs-tim (or one of the nuclear hs-tim constructs). Twenty-five nanograms of a $\beta$-gal reporter gene under a baculoviral immediate early gene promoter were used as a transfection control, and the total DNA amounts per well were kept constant by adding pAct (empty vector only). S2 cells ( 0.75 million per well, in 12 -well plates) were incubated with the DNA-Cellfectin mix in a serum-free medium (Life Technologies). After 3 hr 10\% FBS containing medium was added. In some experiments, cells were heat shocked once every $12 \mathrm{hr}$ for $30 \mathrm{~min}$ at $37^{\circ} \mathrm{C}$. LMB was added to the medium during the heat shocks, at a final concentration of $5 \mathrm{ng} / \mathrm{ml}$. In these cases the cells were allowed to recover in the presence of LMB for $4 \mathrm{hr}$, after which the medium was replaced with fresh LMB-free medium. Cells were harvested $48 \mathrm{hr}$ after transfection, washed in PBS, and lysed in $1 \times$ lysis buffer (Promega). Aliquots of the cell extracts were assayed for $\beta$-galactosidase and luciferase activities (Tropix and Promega, respectively) in a luminometer (Hewlett Packard Top Count). The average ratios of luciferase activity to baseline $\beta$-galactosidase activity were calculated and normalized. Each experimental condition was done in triplicate, and the mean and SEM of three independent experiments were calculated.

Whole-mount immunofluorescence. Populations of developing $\mathrm{per}^{01}$ and $y w$ Drosophila were entrained to light/dark cycles for $4 \mathrm{~d}$ before collection of third-instar larvae. Entrained larvae were collected on ice (in the dark) at times indicated in Results. Whole larval brains were dissected on ice in Schneider's Drosophila cell culture medium supplemented with 10\% FBS and proteosomal inhibitor MG132 [200 $\mu \mathrm{M}$ Z-Leu-Leu-Leu-H (Peptide Institute, Inc.) in DMSO], with or without Leptomycin B (in 70\% methanol; Sigma). Tissue was then incubated with supplemented medium for $1.5-5 \mathrm{hr}$ (as indicated in Results and Figs. 4,5 ) in foil-wrapped 12 -well cell culture plates, with shaking. Brain tissue was then fixed for 20 min with $4 \%$ paraformaldehyde in PBS and washed three times with PBS-Triton X-100 (0.5\%) for 20 min each. Brains were blocked for $1 \mathrm{hr}$ using 10\% normal donkey serum in PBS. To all tissues, anti-TIM (UPR8 1:2500) or anti-PER (UPR34 at 1:500) and rabbit anti-PDF (1:1000) antibodies were added and incubated overnight at $4^{\circ} \mathrm{C}$. The following day the brains were washed three times for $45 \mathrm{~min}$ 
Table 1. Activity rhythms in flies expressing multiple copies of TIM

\begin{tabular}{|c|c|c|c|c|c|}
\hline & Genotype & No. of copies TIM & $n$ (No. of rhythmic) & Average period + SEM & \% Rhythmic \\
\hline \multirow{15}{*}{$\begin{array}{l}\text { Locomotor data from Tim } 4 \text { lines } \\
\text { tim }^{+} / \mathrm{tim}^{+} \text {background }\end{array}$} & \multirow[t]{3}{*}{ Tim 4-1 } & ++ & $36(26)$ & $23.21 \pm 0.21$ & 72.2 \\
\hline & & ++ & $47(42)$ & $23.21 \pm 0.12$ & 89.4 \\
\hline & & $++\mathrm{TT}$ & $49(42)$ & $23.35 \pm 0.10$ & 85.7 \\
\hline & \multirow[t]{3}{*}{$\operatorname{Tim} 4-6$} & ++ & $30(14)$ & $23.67 \pm 0.21$ & 46.7 \\
\hline & & $++\mathrm{T}$ & $22(15)$ & $23.71 \pm 0.09$ & 68.2 \\
\hline & & $++\mathrm{TT}$ & $26(22)$ & $23.71 \pm 0.13$ & 84.6 \\
\hline & \multirow[t]{3}{*}{ Tim 4-7 } & ++ & $12(12)$ & $23.79 \pm 0.14$ & 100 \\
\hline & & $++\mathrm{T}$ & $72(68)$ & $23.60 \pm 0.06$ & 94.4 \\
\hline & & $++\mathrm{TT}$ & $90(81)$ & $23.63 \pm .046$ & 90 \\
\hline & \multirow[t]{3}{*}{ Tim 4-7 × Tim4-1 } & $++\mathrm{TT}$ & $12(9)$ & $24.11 \pm 0.33^{*}$ & 75 \\
\hline & & $++\mathrm{TTT}$ & $54(45)$ & $23.58 \pm 0.10$ & 83.3 \\
\hline & & $++\mathrm{TTTT}$ & $76(70)$ & $23.57 \pm 0.06$ & 92.1 \\
\hline & \multirow[t]{3}{*}{$\operatorname{Tim} 4-7 \times \operatorname{Tim} 4-6$} & $++\mathrm{TT}$ & $16(16)$ & $23.66 \pm 0.19$ & 100 \\
\hline & & + + TTT & $72(65)$ & $23.36 \pm 0.06^{*}$ & 90.3 \\
\hline & & ++ TTTT & $96(76)$ & $23.38 \pm 0.07^{*}$ & 79.2 \\
\hline \multirow{3}{*}{$\begin{array}{l}\text { Locomotor data from Tim } 7 \text { lines } \\
\qquad \operatorname{tim}^{01} / \text { tim }^{01} \text { background }\end{array}$} & \multirow[t]{2}{*}{$\operatorname{Tim} 7-5$} & $\mathrm{~T}$ & $18(14)$ & $24.00 \pm 0.15^{*}$ & 77.8 \\
\hline & & TT & $39(29)$ & $24.22 \pm 0.07^{*}$ & 74.4 \\
\hline & $\operatorname{Tim} 7-4$ & $\mathrm{TT}^{a}$ & $49(38)$ & $24.30 \pm 0.06^{*}$ & 77.6 \\
\hline \multirow[t]{5}{*}{$\operatorname{tim}^{+} /$tim $^{+}$background } & \multirow[t]{3}{*}{$\operatorname{Tim} 7-5$} & ++ & $10(6)$ & $23.33 \pm 0.11$ & 60.0 \\
\hline & & $++\mathrm{T}$ & $16(14)$ & $23.39 \pm 0.09$ & 87.5 \\
\hline & & $++\mathrm{TT}$ & $17(16)$ & $23.09 \pm 0.15$ & 94.1 \\
\hline & \multirow[t]{2}{*}{$\operatorname{Tim}$ 7-4 } & ++ & $22(19)$ & $23.00 \pm 0.19$ & 86.4 \\
\hline & & $++\mathrm{TT}^{a}$ & $43(40)$ & $23.20 \pm 0.06$ & 93.0 \\
\hline
\end{tabular}

Increased TIM has no effect on period length. To measure locomotor activity, flies were placed in locomotor activity monitors under constant conditions at $25^{\circ} \mathrm{C}$ for $7 \mathrm{~d}$. + refers to one copy of endogenous tim. Trefers to one copy of Tim 4 or Tim 7 transgenic tim. An asterisk indicates that the average period of this genotype is significantly longer or shorter than $y w$ control flies (nontransgenic flies from the line in a wild-type background) according to a Student's $t$ test ( $p<$ 0.05 for all). It is clear that these mild differences are not related to transgene dose and are also not consistent from one Tim 4 transgenic line to another.

${ }^{a}$ Because Tim 7-4 was located on the $X$ chromosome, one copy of this transgene was counted as two because of dosage compensation in males.

with rocking. Brains were incubated with secondary antibodies at 1:500 (cyanin 3-conjugated anti-rabbit and fluorescein isothiocyanateconjugated anti-rat; Jackson ImmunoResearch, West Grove, PA) for $1 \mathrm{hr}$ at room temperature. Tissue was then washed for $45 \mathrm{~min}$ three times and mounted with VectaShield (Vector Laboratories).

Sample tissue slides were scored blind. For each head, the total number of visible lateral neurons was counted (on the basis of the PDF stain). These were then given a rating on the basis of the clarity of the morphology and whether the cytoplasmic-nuclear boundary could be determined on the basis of PDF staining alone, with " 0 " being unscorable and "+" being clear. Each + neuron was then scored for TIM or PER visibility and clarity. Only lateral neurons in which most of the TIM or PER signal was clearly visible as more nuclear, more cytoplasmic, or uniformly distributed were counted in the final analysis. TIM and PER localization were reported as percentages of the total number of neurons in these three categories. The initial per ${ }^{01}$ experimental parameters with respect to final DMSO concentration (2-3\%), LMB concentration (20-40 $\mathrm{ng} / \mathrm{ml})$, and incubation time with LMB $(1.5-5 \mathrm{hr})$ were varied over seven independent experiments, with similar results each time. The $y w$ experiments used 3\% DMSO and $40 \mathrm{ng} / \mathrm{ml} \mathrm{LMB}$, incubated for $1.5 \mathrm{hr}$.

Motif search in TIM. The reported TIMELESS sequence (id: TIM_DROME) was scanned for common protein motifs using Motif Scan (http://hits.isb-sib.ch/cgi-bin/PFSCAN). A Prosite scan (http:// us.expasy.org/tools/scanprosite/) was used to scan TIM for occurrences of the leucine-rich consensus sequence for CRM1-exportin-dependent nuclear export $\mathrm{Lx}_{(1-3)} \mathrm{Lx}_{(1-3)} \mathrm{LxL}(\mathrm{V} / \mathrm{I} / \mathrm{M}$ ) (in which " $\mathrm{x}$ " indicates any amino acid) (Yagita et al., 2002).

\section{Results}

Increasing Tim dosage does not shorten circadian period

Some clock genes, most notably per, are dosage sensitive (Smith and Konopka, 1982). Smith and Konopka (1982) showed, through a series of experiments using deletions and duplications of the per locus, that there is an inverse relationship between dosage of per and length of the circadian period. Transgenic manipulations of PER levels yield similar results (Baylies et al., 1987; Chen et al., 1998; Hao et al., 1999). To determine whether TIM levels also affect circadian period length in this manner, we increased copies of tim in flies via two different transgenes. One transgene (Tim 4) consisted of tim cDNA fused to $5 \mathrm{~kb}$ of upstream tim promoter region; the other (Tim 7) consisted of genomic tim also fused to the same promoter region (Ousley et al., 1998). A total of five lines with independent insertions were examined.

Tim 4 has been shown to rescue locomotor rhythms in tim $^{01}$ flies (Ousley et al., 1998) and does not interfere with normal rhythms in a wild-type background (Table 1). In addition, extra copies of Tim 4 in a wild-type background did not significantly shorten period. The Tim 4 transcript, however, fails to display robust mRNA cycling (Wang et al., 2001), indicating that Tim 4 lacks sequences required for appropriate regulation of $t i m$. Thus, there is the possibility that although Tim 4 is producing high levels of TIM protein throughout the head (Fig. 1A), it may not be regulated appropriately enough in the clock-relevant neurons to produce a significant effect. Therefore we also looked at two lines, Tim 7-4 and Tim 7-5, that contain a genomic tim transgene and in which the tim transcript cycles like wild-type tim (Wang et al., 2001). The Tim 7 transgene effectively rescues rhythms in tim $^{01}$ flies (Wang et al., 2001). Flies with additional copies of Tim 7 expressed in a wild-type background have periods of $\sim 23 \mathrm{hr}$, but their nontransgenic $y w$ siblings also have a $23 \mathrm{hr}$ period, so the shorter periods cannot be attributed to high TIM expression (Table 1). Western analysis of Tim 4 and Tim 7 lines confirmed that TIM is present at higher than wild-type levels (Fig. $1 A, B$ ). These data suggest that the function of TIM in the clock is dosage insensitive, which supports previous findings that decreasing tim dosage also does not affect period length (Rothenfluh et al., 2000b).

PER and TIM form a heterodimer in a presumably $1: 1$ ratio (Zeng et al., 1996). Because PER is thought to require TIM as a partner both for stability and to enter the nucleus to effect feed- 


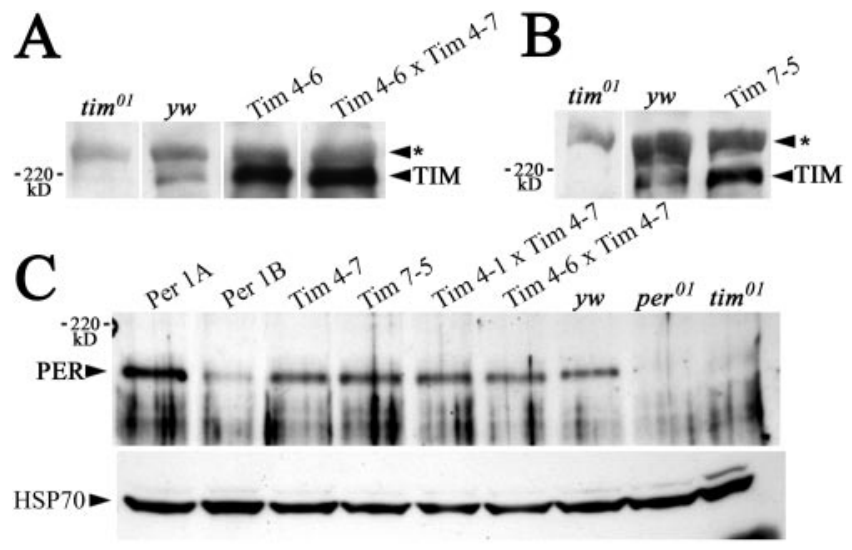

Figure 1. TIM and PER levels in overexpression lines. Protein extracted from adult heads was separated on a $6 \%$ polyacrylamide gel. TIM levels are increased over wild-type ( $y w)$ levels in $\operatorname{Tim} 4(A)$ and Tim $7(B)$ lines collected at ZT 21. Spaces indicate noncontiguous lanes from the same blot. Asterisk indicates a common nonspecific band, used here as a loading control. All Tim 4 and 7 lines were tested, but only one representative line each is shown here for simplicity. In C, flies were collected at an earlier time point, ZT 16.5. PER levels are unaltered in Tim 4 (lanes 3, 5, and 6) and Tim 7 (lane 4) overexpression lines. PER is only mildly increased in Per 1-A and decreased in Per 1-B. Surprisingly, Per 1-B generally has a stronger behavioral phenotype than Per 1-A (Table 2), which probably indicates that Per 1-B has stronger negative feedback (see Discussion). HSP 70 (bottom blot) was used as a loading control. Another analysis of PER levels at ZT 21 showed similar results.

back (Vosshall et al., 1994; Price et al., 1995; Saez and Young, 1996), we hypothesized that it is not PER that is the state variable in this system, but the PER-TIM heterodimer. Thus, changes in PER that increase or decrease the length of the circadian period in flies do so by decreasing or increasing PER-TIM levels; however, on the basis of the following data we infer that TIM is present in excess and thus not a limiting factor in the Drosophila clock: (1) TIM is present in molar excess of PER for part of the night and is present in PER-immunodepleted extracts (Zeng et al., 1996); (2) the tim promoter shows an approximately threefold higher transcription rate than the per promoter (So and Rosbash, 1997); and (3) alterations of TIM levels alone do not alter period length (Rothenfluh et al., 2000a) (data presented here). If TIM is in excess and PER is the limiting factor, we would expect alterations in TIM levels to affect period length only if PER levels are also increased. Despite the increased TIM levels in our Tim 4 and Tim 7 transgenic lines, we did not see altered PER levels over wild type (Fig. 1C), which explains the lack of a short-period phenotype. It might be possible, however, by transgenically manipulating PER levels, to increase PER to the point at which TIM becomes limiting. When Smith and Konopka (1982) increased per dosage via duplications, they found that they never shortened the period of the clock by $>1.5-2$ hr even with five copies of endogenous PER. We hypothesized, therefore, that in the Smith and Konopka study (1982) TIM became limiting when PER levels were increased, and thus the period could not be shortened beyond a certain point.

We intended, therefore, to raise both PER and TIM levels together. To this end, we expressed a per cDNA under control of the same tim promoter region used in the Tim 4 and Tim 7 lines because tim appeared to be the stronger promoter (So and Rosbash, 1997). The Per 1 transgenes rescue rhythms in per $^{01}$ flies, with a period that is $1-2 \mathrm{hr}$ shorter than wild type (Table 2 ), as would be expected from lines that overproduce PER. Per 1 also shortened period in a wild-type background, the Per 1-B insertion generally more so than Per 1-A. Two copies of the Per 1-B transgene shortened period to $22 \mathrm{hr}$ and also decreased the proportion of rhythmic flies in the line.
We then crossed Tim 4 flies to Per 1 flies with the goal of increasing both PER and TIM together. If TIM becomes limiting at high PER concentrations, then increasing both proteins should shorten the period even further. As is evident from the locomotor data in the second half of Table 2, additional copies of Tim 4 into the Per 1 lines did not shorten period any further than Per 1 alone. Statistical analysis of all groups by two-way ANOVA revealed a significant effect of the Per 1 transgenes on period, but no significant effect of Tim 4. We conclude, therefore, that the clock is relatively insensitive to tim dosage, and TIM is generally present in excess for most of the night (until levels decline around dawn). Manipulation of per levels can only shorten the period of the clock so far $(\sim 2 \mathrm{hr})$ before either another clock factor becomes limiting or some other critical regulatory mechanism is overcome and the molecular cycle runs into arrhythmia.

Immunohistochemistry of adult heads from Per-1 lines revealed PER expression in cells that normally express PER and TIM (data not shown). Surprisingly, Western blot analysis showed only a small increase in steady-state PER levels in the Per $1 \mathrm{~A}$ line and a decrease in the Per1B line (Fig. 1C). The lack of a robust increase of PER levels in these lines could be attributable to amplified negative feedback by the overproduced PER, which would ultimately result in decreased protein levels and an accelerated cycle. There may also be differential per expression in peripheral oscillators versus $\mathrm{LN}_{\mathrm{v}} \mathrm{s}$, such that whole-head extracts show lower than expected PER levels. In these lines, however, PER is driven by the same promoter that drives elevated TIM expression in Tim 4 and TIM 7 lines (Fig. $1 A, B$ ). Therefore we favor the hypothesis that excess PER is negatively regulating its own expression but TIM is not (see Discussion).

\section{TIM cannot repress dCLK-CYC-mediated transcription unless PER is present}

It is thought that PER must bind to TIM to gain entry into the nucleus and effect transcriptional changes (Vosshall et al., 1994; Saez and Young, 1996). Both proteins contain an NLS as well as a CLD and removing the CLD from either protein frees it from the dimerization requirement for nuclear entry (Saez and Young, 1996); however, PER does not require TIM to exert its effects on transcription. Rothenfluh et al. (2000a) demonstrated that deletion of the CLD allows PER to repress dCLK-CYC-activated transcription in the absence of TIM, and flies expressing this altered PER display a high degree of arrhythmicity (Rothenfluh et al., 2000a). Also, they showed that maximal transcriptional repression in flies occurs during the time that TIM has degraded and only PER remains (Rothenfluh et al., 2000a). Shafer et al. (2002) recently reported that some PER is visible in the nucleus 3-4 hr ahead of TIM in the behavioral clock relevant $\mathrm{LN}_{\mathrm{vs}}$, and they noted that the time of PER nuclear entry coincides with the onset of transcriptional repression.

Thus, PER clearly has a role in circadian transcription repression, but the role of TIM in this process is unknown. Some evidence that TIM might have a role in transcription came from a Drosophila mutant defective in PER feedback, per $\Delta$ C2. dCLKCYC-activated gene expression remains high in these lines even during the late evening, but a light pulse that degrades TIM causes a further increase in dCLK-CYC-activated transcripts (Schotland et al., 2000). TIM has also been shown to disrupt dCLK and CYC binding to a circadian E-box in vitro (Lee et al., 1999). Efforts to confirm a transcriptional role of TIM alone in cell culture or Drosophila, however, were hampered by the mechanisms that sequester TIM in the cytoplasm in the absence of PER. To address whether TIM can directly regulate transcriptional activity, with- 
Table 2. Activity rhythms of flies expressing PER and TIM

\begin{tabular}{|c|c|c|c|c|c|}
\hline & Genotype & No. of copies PER & $n$ (No. of rhythmic) & Average period + SEM & \% Rhythmic \\
\hline \multirow{4}{*}{$\begin{array}{l}\text { Locomotor data from Per } 1 \text { lines } \\
\text { per }^{01} / \mathrm{per}^{01} \text { background }\end{array}$} & Per 1-A/+ & $P$ & $46(24)$ & $21.69 \pm 0.84^{*}$ & 52.2 \\
\hline & Per 1-A/Per 1-A & PP & $39(24)$ & $22.38 \pm 0.11^{*}$ & 61.5 \\
\hline & Per 1-B/+ & $P$ & $16(14)$ & $22.43 \pm 0.10^{*}$ & 87.5 \\
\hline & Per 1-B/Per 1-B & PP & $58(12)$ & $22.54 \pm 0.18^{*}$ & 20.7 \\
\hline \multirow[t]{5}{*}{ per $^{+} /$per ${ }^{+}$background } & $+/+$(Per 1-A siblings) & ++ & $28(26)$ & $23.58 \pm 0.09$ & 92.9 \\
\hline & Per 1-A/+ & $++P$ & $85(77)$ & $22.39 \pm 0.05^{*}$ & 90.6 \\
\hline & $+/+$ (Per 1-B siblings) & ++ & $32(29)$ & $23.53 \pm 0.07$ & 90.6 \\
\hline & Per 1-B/+ & $++P$ & $72(67)$ & $22.68 \pm 0.07^{*}$ & 93.1 \\
\hline & Per 1-B/Per 1-B & $++P P$ & $32(10)$ & $22.10 \pm 0.16^{*}$ & 31.3 \\
\hline \multirow{8}{*}{$\begin{array}{l}\text { Locomotor data from Per } 1 \times \text { Tim } 4 \text { lines } \\
\text { per }^{+} / \text {per }^{+}, \text {tim }^{+} / \text {tim }^{+} \text {Background }\end{array}$} & Per 1-A × Tim 4-7 & PT & $97(82)$ & $22.79 \pm 0.07^{*}$ & 84.5 \\
\hline & & P TT & $122(84)$ & $22.68 \pm 0.06^{*}$ & 68.9 \\
\hline & & PPT & $19(12)$ & $22.38 \pm 0.24^{*}$ & 63.2 \\
\hline & & PPTT & $20(9)$ & $22.28 \pm 0.15^{*}$ & 45.0 \\
\hline & Per 1-B × Tim 4-7 & PT & $52(49)$ & $22.65 \pm 0.06^{*}$ & 94.2 \\
\hline & & P TT & $78(66)$ & $22.27 \pm 0.07^{*}$ & 84.6 \\
\hline & & PPT & $64(8)$ & $21.69 \pm 0.25^{*}$ & 12.5 \\
\hline & & PPTT & $62(8)$ & $21.94 \pm 0.31^{*}$ & 12.9 \\
\hline
\end{tabular}

Increased PER, but not TIM, affects period length. To measure locomotor activity, flies were placed in locomotor activity monitors under constant conditions at $25^{\circ} \mathrm{C}$ for $7 \mathrm{~d}$. The endogenous per gene is on the $\mathrm{X}$ chromosome. In males, per is dosage compensated, such that the effect of one per copy in males is equivalent to two in females. We count it here as two copies. P represents one copy of Per 1; T represents one copy of Tim 4-7. + represents one copy of endogenous per. Although it is not indicated in the bottom of the Table with a + , all flies in Per $1 \times$ Tim 4 lines had endogenous per and tim. An asterisk indicates that the average period of this genotype is significantly different from its nontransgenic siblings ( $p<0.05$; Student's $t$ test). Note: The wild-type Per 1-A stock developed a spontaneous independent mutation that significantly reduced the viability of this line, making it difficult to obtain reliable locomotor data from homozygous flies. There was a significant effect of PER on period length in the Per $1 \times \operatorname{Tim} 4$ lines when measured by two-way ANOVA $(p<0.000)$, but not of TIM in the same lines $(p=0.53)$. Thus, additional TIM in the circadian system does not significantly alter period.

out PER, we modified TIM to increase its nuclear localization either by deleting the CLD region (hs-tim $\Delta^{C L D}$ ) or by adding a heterologous NLS (hs-tim ${ }^{+N L S}$ ). We then tested the ability of these modified TIM proteins to affect in vitro transcription in the absence of PER.

dCLK/CYC-mediated transcriptional activation in S2 cells was assayed using a per E-box reporter system developed by Darlington et al. (1998). We wanted to study the effect of nuclear TIM on dCLK/CYC activity, with and without PER. Using a luciferase reporter (E-box luc) to measure transcription levels, we cotransfected into S2 cells dCLK and one of the three inducible versions of tim (Fig. $2 A$ ): hs-tim, hs-tim $\Delta^{C L D}$, and hs-tim ${ }^{+N L S}$. The activation by dCLK alone ( $\sim 100$ - to 200 -fold) was normalized to $100 \%$. Both full-length and modified forms of TIM interact with PER to repress transcription at concentrations in which neither protein can repress alone, showing that the two modifications did not interfere with the ability of TIM to enable repression (Fig. 2 B, left). A 10-fold increase of PER (which presumably enables PER to overcome cytoplasmic retention mechanisms) allows it to repress transcription without cotransfection of TIM. In contrast, when likewise increased 10 -fold, none of the versions of TIM could repress transcription (Fig. $2 B$, right). Strangely, high concentrations of TIM caused a two- to threefold increase in the luciferase reporter signal. This could not be attributed to a general increase in transcription caused by the heat shock, because it also occurred when TIM was expressed at high levels via an actin promoter (data not shown). Also, the addition of $100 \mathrm{ng}$ of PER at this higher concentration resulted in the expected repression equivalent to high concentrations of PER alone (data not shown). We propose several explanations for this unexpected effect of TIM in Discussion.

There are two possible explanations for lack of repression by TIM alone in our first transcription assay. The first is that TIM requires PER to exert a negative effect on transcription. A second possibility is that these TIMs are not nuclear, despite the modifications. To test this, we expressed the aforementioned modified TIM proteins in S2 cells and visualized the subcellular localization of TIM through immunofluorescence (Fig. 3A). Two hours after induction of TIM expression by heat shock, cells were scored for relative staining intensities in the nucleus and cytoplasm. $\mathrm{TIM}^{+\mathrm{NLS}}$ protein was visible in the nucleus $25 \%$ of the time (Fig. $3 B$ ). Of the cells expressing TIM $\Delta^{\mathrm{CLD}}, 5 \%$ demonstrated nuclear staining, and there was a significant increase (30\%) in the number of uniformly stained cells. In contrast, full-length TIM was almost entirely cytoplasmic. It was shown previously that $\mathrm{TIM} \Delta^{\mathrm{CLD}}$ is more nuclear when assayed $4 \mathrm{hr}$ after induction (Saez and Young, 1996, their Fig. 3); however, similarly modified PER (CLD deleted) is nuclear after $2 \mathrm{hr}$ (Saez and Young, 1996, their Fig. 2). We suggest that there is another mechanism differentially regulating the nuclear entry of TIM that is not necessarily acting through the CLD.

\section{Subcellular localization of TIM is regulated in part by active nuclear export}

In mammals, mCRY and mPER were also thought to require heterodimerization for PER nuclear entry (Kume et al., 1999). A recent study by Yagita et al. (2002), however, revealed that mPER2 is regulated by nuclear export and does not require dimerization with mCRY for nuclear entry but only for nuclear accumulation. It seemed quite possible, given our results with the TIM proteins that were still essentially cytoplasmic despite the modification to transport them to the nucleus, that Drosophila TIM is also actively exported from the nucleus. Therefore, we made use of the specific nuclear export inhibitor LMB to block nuclear export. LMB binds to the nuclear export protein CRM1, inhibiting its ability to bind to proteins containing the most common nuclear export signal (Nishi et al., 1994; Henderson and Eleftheriou, 2000). The results, shown in Figure 4, $A$ and $B$, were dramatic; within $2 \mathrm{hr}$ after induction, unmodified TIM localized to the nucleus in a dose-dependent manner, becoming almost entirely nuclear at a concentration of $2 \mathrm{ng} / \mathrm{ml}$ of LMB. Even the smallest dose of $1 \mathrm{ng} / \mathrm{ml}$ significantly increased nuclear TIM.

Because the previous experiments were performed in embryonic cultured cells instead of clock-relevant lateral neurons, we decided to repeat this test in flies lacking functional endogenous 


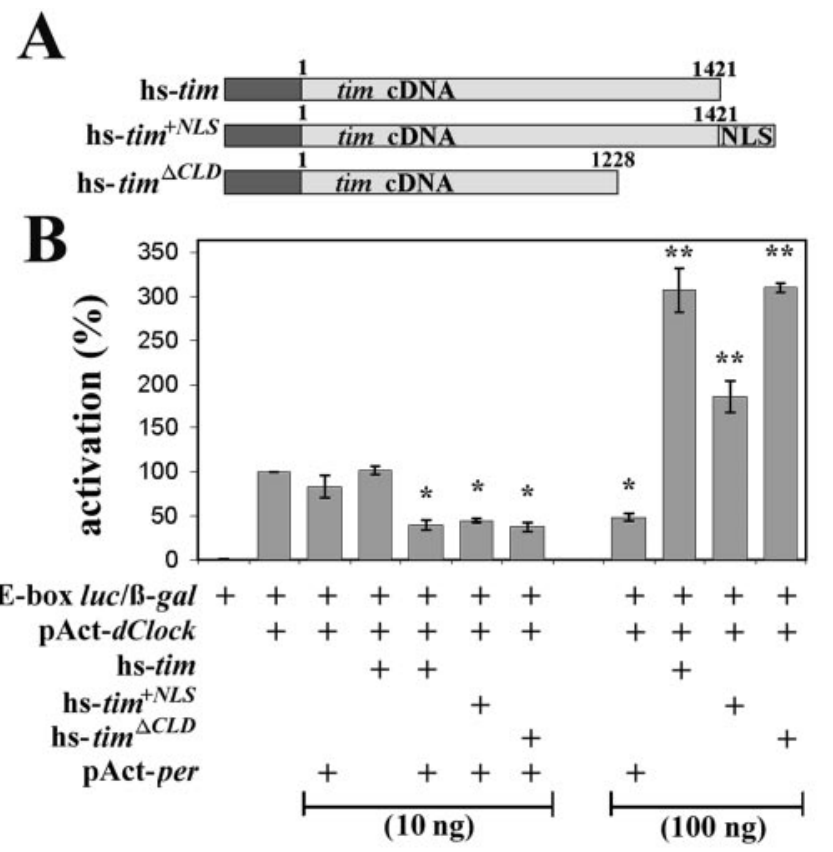

Figure 2. TIM alone cannot repress dCLK-CYC-mediated transcription of a circadian E-box reporter. An inducible tim gene was modified to permit nuclear entry of TIM in the absence of PER, as shown in $A$ (see Results). Although the modifications still allowed TIM and PER together to repress transcription (asterisk indicates signal is significantly less than $\mathrm{pAct-dClk}$ alone; $p<$ 0.01 ; $t$ test), even high doses of nuclear TIM cannot repress transcription without PER. Surprisingly, TIM alone appeared to activate transcription (double asterisk indicates that signal is significantly greater than dCLK activation alone; $p<0.01 ; t$ test). Differences among hs-tim, $\mathrm{tim}^{+N L S}$, and tim $\Delta C L D$ are not significant. Drosophila S2 cells were transiently transfected with two reporter genes (E-box luc and i.e. $\beta$-gal), and a combination of pAct- $d C l k$, full-length or mutated hs-tim, and pAct-per, as indicated by + . After $48 \mathrm{hr}$, cells were harvested and assayed for luciferase and $\beta$-gal activity. Data were normalized to $\beta$-gal expression to control for transfection efficiency. Results are presented as percentage of full activation seen in the $d C l k$ alone condition and expressed as averages ( \pm SEM) of three triplicate measures. Two other identical independent experiments showed similar results.

PER. In per $^{01}$ flies, TIM accumulates to high levels in the dark (Myers et al., 1996; Zeng et al., 1996). We therefore entrained per $^{01}$ larvae to an LD schedule and collected them at ZT 20-21, a time during which TIM has been shown to be nuclear in adult wild-type lateral neurons (Shafer et al., 2002). The CNS was dissected and incubated for $1-5 \mathrm{hr}$ in medium containing LMB. The whole-mounts were then fixed and double labeled with antibodies to PDF and TIM. PDF, a peptide normally transported to axon terminals and thus cytoplasmic in lateral neurons (Helfrich-Forster, 1995; Shafer et al., 2002), was used to locate the lateral neurons in the optic lobes of developing larvae (Kaneko et al., 1997) as well as to determine the location of the cytoplasm. TIM localization was scored as described previously. In control per $^{01}$ larvae, most of the TIM (78\%) was found to localize to the cytoplasm as reported previously (Myers et al., 1996). In experiments where LMB blocked nuclear export, however, there was a significant increase in TIM localization in the nucleus. Samples of TIM fluorescence (red) are shown with their corresponding PDF signals (green) in Figure 4C. We present the pooled results of seven independent experiments in graphic form in Figure $4 D$. Thus, in both cultured cells and larval clock cells, TIM is imported into the nucleus in the absence of PER and exported again through CRM1-dependent mechanisms.
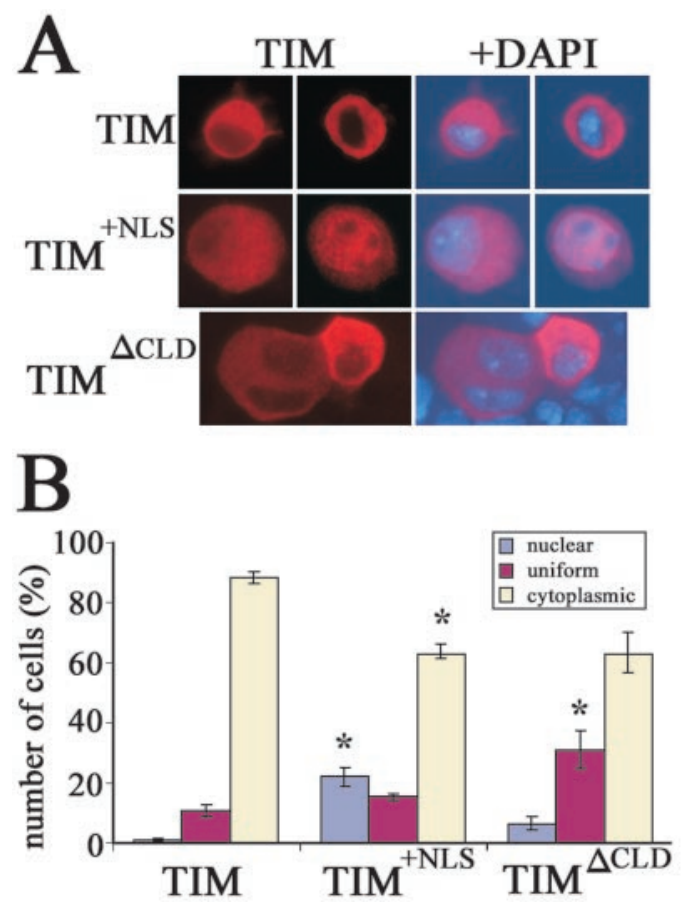

Figure 3. TIM localizes predominantly to the cytoplasm in transiently transfected S2 cells despite modifications to increase nuclear entry. Shown in $A$ are representative samples of the subcellular distribution patterns of TIM with and without modifications to increase nuclear entry. Quantification of three independent experiments $(B)$ revealed that although the modifications do tend to increase TIM distribution in the nucleus, the effect is not as robust as we expected, indicating that there may be alternative mechanisms acting on TIM localization. S2 cells transfected with $500 \mathrm{ng}$ of full-length or modified hs-tim (in 6-well plates) were fixed $2 \mathrm{hr}$ after heat shock induction of TIM expression. TIM localization was scored as mostly nuclear, mostly cytoplasmic, or uniformly distributed (left columns, in red), and nuclei were visualized with DAPI (right columns, overlaid against TIM signal). Data are presented as mean percentages of cells in each category ( \pm SEM). Asterisk indicates that this group is significantly different from full-length TIM for that subcellular localization ( $p<0.01$; one-way ANOVA).

\section{Nuclear export of TIM is affected by PER}

LMB has no significant effect on the localization of PER alone in S2 cells (data not shown); however, the subcellular localization of TIM in S2 cells changed after coexpression with PER (Fig. 5A,B). Although TIM alone was completely nuclear when export was blocked, it was significantly more cytoplasmic when PER was present. The effect is PER specific; equivalent amounts of $\beta$-galactosidase coexpressed with TIM did not have any effect on TIM localization with or without LMB (data not shown). Note that at this time point ( $2 \mathrm{hr}$ after induction), PER and TIM were also still mostly cytoplasmic in the work of Saez and Young (1996), indicating that the nuclear expression of the heterodimer is normally delayed even in S2 cells. Because TIM alone (with LMB) is nuclear at $2 \mathrm{hr}$, we infer that PER sequesters TIM and delays the nuclear entry of the heterodimer (see Discussion).

Our per ${ }^{01}$ larval data led us to hypothesize that PER is necessary for TIM nuclear retention (as opposed to its transport to the nucleus, as originally thought). The cell culture data indicated that PER could also retain TIM in the cytoplasm, suggesting that the effects of PER on TIM could be different under different conditions. To determine the effect of PER on TIM in vivo, we repeated our larval CNS assay, this time looking at both TIM and PER localization in $y w$ larvae. Because these flies have a working clock, and thus PER and TIM localization varies during the course of the evening, we looked at two different time points, ZT 15.5 and ZT 19.5 ( $n=55$ and 261 LNs scored, respectively). Our 
results are summarized in Figure 5, $C$ and $D$. At both time points, LMB treatment resulted in an increase in nuclear expression of TIM and also of PER, indicating that TIM does influence PER localization to some extent. The effect of blocking nuclear export on nuclear expression of both proteins was greater at ZT 19.5, perhaps because PER is more nuclear at this time point (Shafer et al., 2002). Thus, as in the S2 cells, PER most likely has a profound effect on the subcellular localization of TIM.

TIM does not repress dCLK-CYCmediated transcription even when its export from the nucleus is blocked Having shown that the addition of LMB causes a dramatic increase in nuclear TIM, we repeated the transcription assay described in Figure 2, modified to block nuclear export of TIM. S2 cells were transiently transfected with the various reporter constructs, pAct- $d$ Clock and hs-tim or pAct-per, or both. TIM expression was again induced via heat shocks, this time in the presence of LMB at a high dose shown to result in nuclear TIM (10 ng/ml; see Materials and Methods for details). Figure 6 shows the result of a representative experiment performed in triplicate, normalized to $100 \%$ activation by dCLK. Because we used smaller doses of TIM, we did not see the transcriptional activation that is evident at larger doses. The addition of $\mathrm{LMB}$ did not result in any repressive effect of TIM on transcription. Also, LMB had no effect on the ability of PER and TIM together to repress transcription.

\section{Discussion}

We used behavioral and genetic assays to analyze the role and regulation of TIM in the circadian clock, on the basis of predictions of the current clock model. Our results show that the clock is insensitive to tim dosage. We also present evidence that TIM alone cannot repress dCLK-CYC-mediated transcription of an E-box reporter in S2 cells, despite a report that TIM disrupts the DNA-binding ability of dCLK and CYC in vitro (Lee et al., 1999). Finally, we present evidence that TIM does not require dimerization with PER for nuclear entry, although PER is involved in TIM nuclear accumulation and retention.

We found that increased levels of TIM do not shorten period length. This is not surprising, because Zeng et al. (1996) found that TIM is present in molar excess of PER for part of the night. TIM is present in PER-immunodepleted extracts (Zeng et al., 1996), the tim promoter is stronger than the per promoter (So and Rosbash, 1997), and decreasing tim dosage does not affect period length (Rothenfluh et al., 2000b). Thus, there is ample evidence that TIM levels exceed PER levels for most of the night (until dawn, when TIM levels drop), and alterations of TIM levels have little effect on PER function.

Surprisingly, PER levels in whole-head extracts were not

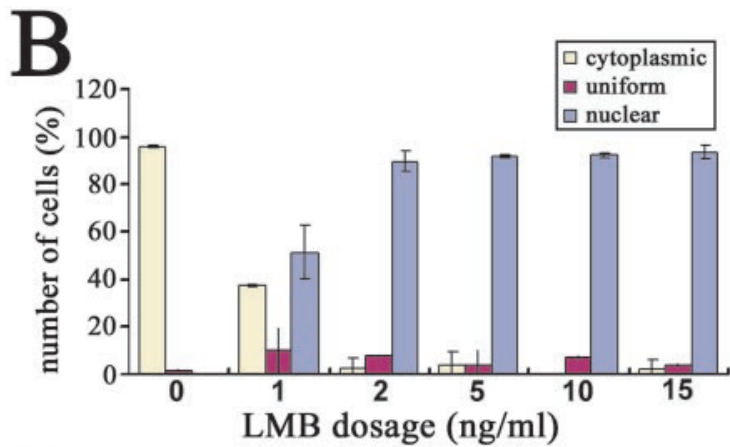

D

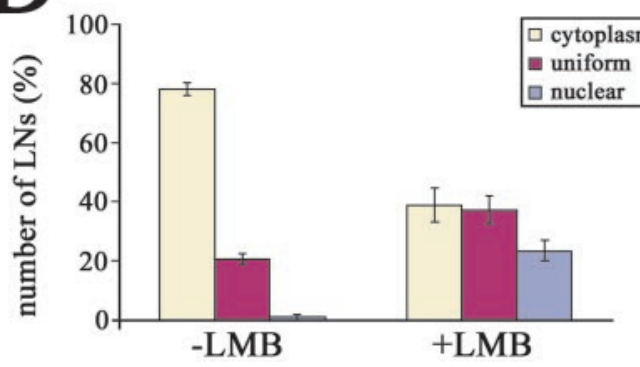

cytoplasmic uniform

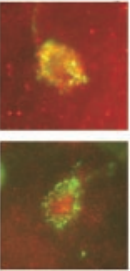

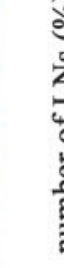

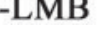

+LMB

Figure 4. Nucleocytoplasmic shuttling of TIM in cell culture and $p e r^{01}$ lateral neurons. Localization of TIM was measured in the presence of a nuclear export blocker (LMB) in transiently transfected S2 cells and larvae. In both cases, blocking nuclear export led nucleus and actively exported. A shows representative examples of TIM subcellular localization in S2 cells. Five hundred nanogram of hs-tim (full-length only) were transfected, expressed, and scored as described in Figure 3, with one exception: LMB (10 ng/ml) xpression occurs extremely rapidly, even within 30 min after induction (data not shown). In $B$, TIM localization was quantified as TIM (red) and PDF (green overlay) signals in per ${ }^{01}$ larval LNs. TIM is cytoplasmic in per ${ }^{01}$ flies (above), and LMB addition significantly increased the proportion of TIM found in the nucleus in per $^{01}$ flies (below) in seven independent experiments, quantified in $D$ 等 $\mathrm{ng} / \mathrm{ml}$ ) and supplemented with MG132 (a proteasomal inhibitor) to block TIM degradation. After the incubation period, the tissue slightly to enhance the signal over the background. As with the cell culture quantification data are presented as percentages of which most of the TIM signal was nuclear, cytoplasmic, or uniformly distributed. Only LNs with clear cytoplasmic-nuclea

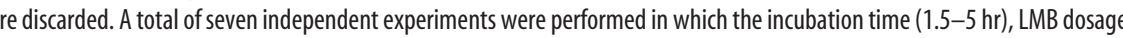
$(20-40 \mathrm{ng} / \mathrm{ml})$, and DMSO (1-3\%) concentration were varied, with similar results.

markedly increased when overexpressed via the tim promoter, despite the dramatic short-period phenotypes (Fig. 1C, Table 2). This is most likely attributable to elevated negative feedback of PER onto the tim promoter, which would result in decreased steady-state levels of protein despite increased synthesis. Feedback is presumably required for the shorter period, because we have noticed that overexpression of per by heterologous promoters, such as actin and elav, actually causes a lengthening of period as well as arrhythmia (Yang and Sehgal, 2001), whereas overexpression of per with an E box-containing promoter leads to shorter periods (Hao et al., 1999). This may also explain why the period length cannot be shortened by PER overexpression beyond 21.5-22 hr without loss of protein oscillations and overt behavioral rhythms (Smith and Konopka, 1982; Yang and Sehgal, 2001; this study). Beyond this point the clock may not be able to compensate for the increased PER feedback. Thus, the notion that higher levels of PER shorten period, and vice versa, may be true only when per can inhibit its own expression. Otherwise, increased PER lengthens period (because of prolonged protein expression) or causes arrhythmia. On the other hand, TIM levels 


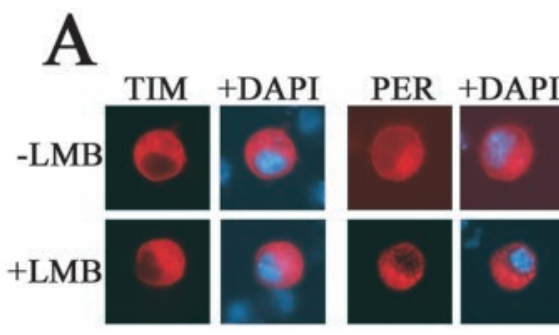

B

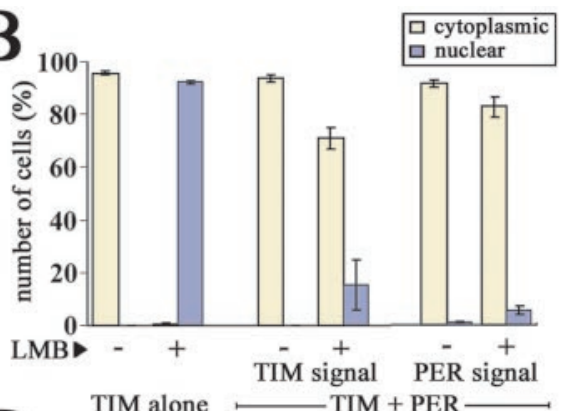

C

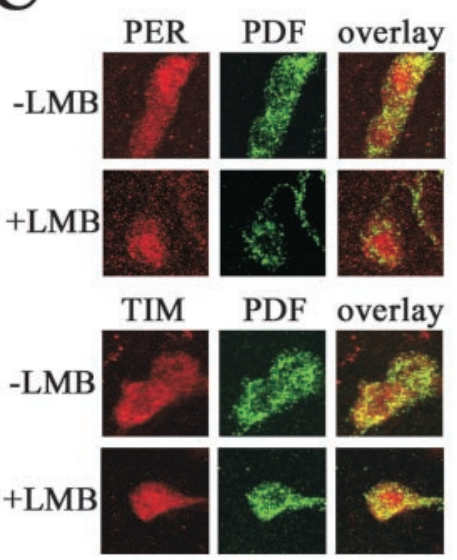

D
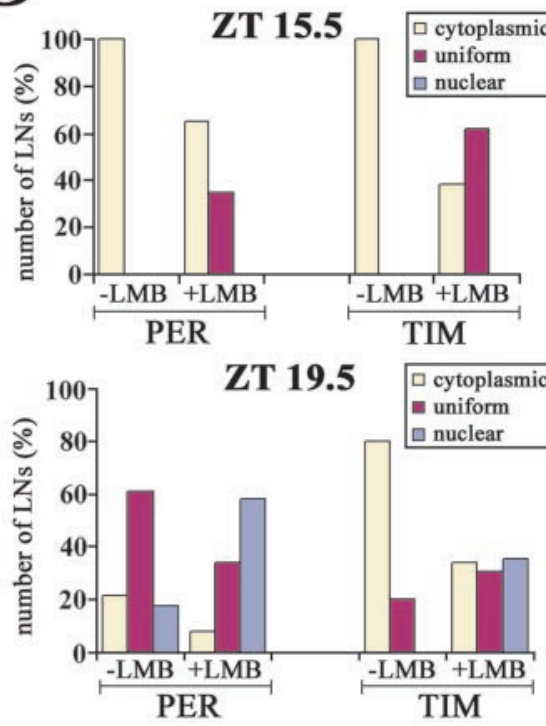

Figure 5. PER alters TIM localization in vitro and in vivo. PER sequesters TIM in the cytoplasm of S2 cells during LMB treatment, indicating that the presence of PER inhibits TIM from entering the nucleus when PER is cytoplasmic. In yw larvae, however, there is still a slight trend toward more nuclear expression of TIM and PER when nuclear export is blocked. The trend is more pronounced when PER is already more nuclear. A shows representative examples of $\mathrm{S} 2$ cells transiently transfected with $500 \mathrm{ng}$ each of hs-tim and pAct-per. Quantification of TIM and PER localization is presented in B, scored as described in Figure 3. S2 cells were treated with LMB during heat shock as described in Figure 4, fixed $2 \mathrm{hr}$ after induction, and stained for either TIM (left, red) or PER (right, red) and DAPI (blue overlay). Data in B were pooled from two independent experiments, and in each experiment 100-300 cells per group were scored. ( presents examples of PER (top) and TIM (bottom) signals in yw larvae, collected at ZT 19.5, incubated with LMB or vehicle for $1.5 \mathrm{hr}$. At this time point, PER is more nuclear. The sample cells show an example of nuclear and uniform PER distribution without LMB and nuclear distribution with LMB. TIM is normally still cytoplasmic, as shown in the (-)LMB example, but there is a definite trend toward nuclear localization when export is blocked by LMB (bottom). PER localization is also affected, as seen in the quantifications of two independent experiments (D). Whole brains from yw third-instar larvae were dissected at ZT $20-21$ and incubated for $3 \mathrm{hr}$ in medium containing (bottom) or lacking (top) LMB (40 ng/ml) and supplemented with MG132 (a proteasomal inhibitor) to block TIM degradation. After the incubation period, the tissue was fixed and probed with antibodies to TIM or PER (visualized with Cy3-conjugated secondary antibodies, red) and compared with the cytoplasmic PDF signal detected with FITC (green). All LNs were scored blind as described in Figure 4.

were increased in the TIM overexpression lines, perhaps because TIM does not directly effect negative feedback (discussed below).

In two different experimental designs, nuclear versions of TIM caused no repression of transcription without coexpression of PER. Our behavioral data also indicate that, unlike PER, higher levels of TIM apparently cannot feed back to repress transcription and alter period length (Lee et al., 1999). This was contrary to our prediction based on the light-induced increase of per transcripts in flies expressing a mutant PER (Schotland et al., 2000). The assumption was that the light induced degradation of TIM relieved the repression of per transcription. There are alternative explanations, however, such as an undescribed transcriptional role of dCRY, which is also degraded by light (Lin et al., 2001). Mammalian CRY plays an active role in clock feedback (Kume et al., 1999), as do some zebrafish CRYs (Kobayashi et al., 2000; Hirayama et al., 2003). In Drosophila, CRY interacts directly with

PER (Rosato et al., 2001) and is a functional component of peripheral clocks (Ivachenko et al., 2001; Krishnan et al., 2001). It is predominantly peripheral head clocks that are reported by the RNase protection assays used by Schotland et al. (2000).

The activating effect of TIM on the luciferase signal was surprising (Fig. 2). This effect is independent of heat shock and abrogated by addition of PER (data not shown). TIM has been shown to promote RNA stability, but this is specific to per RNA and requires the presence of functional PER protein (Suri et al., 1999). We identified a glutamine-rich region in TIM (position 397-412) using Motif Scan; these transcriptional activation domains are found in a number of Drosophila transcription factors, including Sp1 (Gill et al., 1994; Roberts, 2000). This raises an intriguing possibility for a PERindependent role of TIM in the upregulation of other clock-controlled genes during the late evening (Claridge-Chang et al., 2001; McDonald and Rosbash, 2001; Ceriani et al., 2002; Lin et al., 2002); however, we believe that this "activation" more likely reflects a positive effect of TIM on $d C l k$ RNA (Bae et al., 1998; Lee et al., 1998), either by increasing $d C l k$ RNA stability or perhaps by derepressing the endogenous $d C l k$ gene by blocking VRILLE repression (Glossop et al., 1999, 2003; Cyran et al., 2003).

The effect of exogenous PER on TIM in the presence of LMB indicates a strong influence of PER on TIM localization. We note that PER and TIM were cytoplasmic at earlier time points (shortly after they are coexpressed) in the Saez and Young (1996) study. Thus, in S2 cells, as in flies, nuclear entry of the heterodimer is delayed. In the LMB experiments, TIM is almost immediately nuclear without PER, indicating that TIM nuclear import is not delayed in the absence of PER. A likely model is that PER sequesters TIM in the cytoplasm until PER itself is transported actively to the nucleus. Thereafter, it retains TIM in the nucleus. These results are supported by our in vivo data, where we found that the effect of LMB on PER and TIM localization in larval lateral neurons varied depending on the time point chosen (and the location of PER). Thus, PER may be the dominant determinant of PER-TIM localization and thus the more likely candidate for mechanisms regulating nuclear entry.

TIM, however, must also play a role in this process the basis of the following lines of evidence: (1) a PER- $\beta$ Gal fusion protein is cytoplasmic in tim $^{01}$ flies, although nuclear in wild type (Vosshall et al., 1994); (2) the presence of TIM promotes nuclear expression of PER at later time points in S2 cells (Saez and Young, 1996); (3) per $^{L}$ mutants, defective in TIM binding, also display a delay in nuclear entry (Curtin et al., 1995; Gekakis et al., 1995); (4) over- 


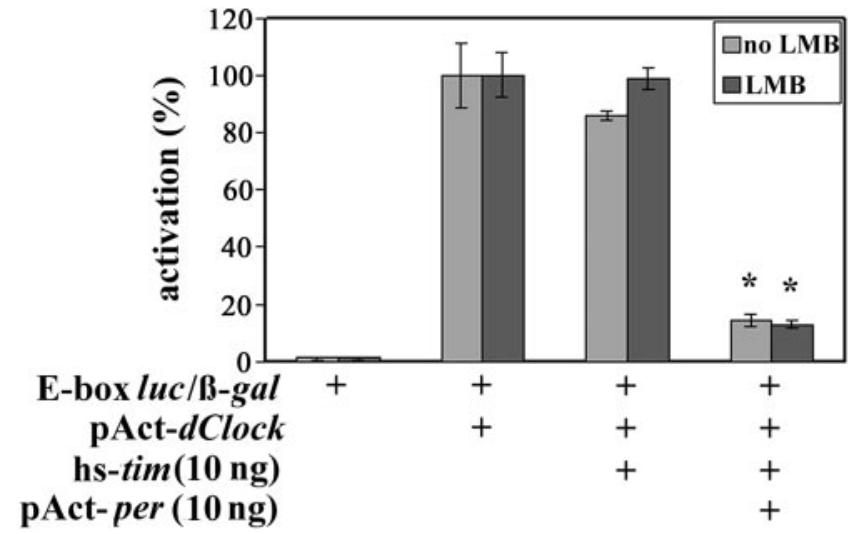

Figure 6. TIM alone does not repress dCLK-CYC-mediated transcription even when its nuclear export is blocked. LMB treatment had no significant effect on either TIM repression or $\mathrm{TIM}+$ PER repression. After transient transfection of $\mathrm{S2}$ cells (described previously in Fig. 2), cells were heat shocked for 30 min every $12 \mathrm{hr}$, in medium supplemented with $5 \mathrm{ng} / \mathrm{ml} \mathrm{LMB}$. Four hours after induction, the medium was replaced with LMB-free medium. After $36 \mathrm{hr}$, cells were collected and analyzed for luciferase and $\beta$-gal expression as described in Figure 2 . Results are expressed as averages ( \pm SEM) of three triplicate measures. Two other identical independent experiments showed similar results. Asterisk indicates that the amount of activation is significantly less than activation by dCLK alone ( $p<0.01 ; t$ test).

expression of shaggy ( $\mathrm{sgg}$ ), a kinase that phosphorylates TIM, causes a $2 \mathrm{hr}$ advance in PER nuclear entry (Martinek et al., 2001); and (5) LMB, which does not affect PER localization directly, increases PER localization to the nucleus, presumably via the PER interaction with TIM (Fig. 5). Interestingly, PER has been shown to interact with TIM via the TIM NLS, whereas TIM interacts with the PER CLD (Saez and Young, 1996). We suggest that these interactions contribute to the delay in nuclear entry of the heterodimer, which eventually becomes nuclear through active regulation-modification of PER. In light of the recently reported differential timing of PER and TIM nuclear entry (Shafer et al., 2002), a possible explanation is that TIM and PER enter the nucleus as a dimer, but initially TIM is actively exported from the nucleus. A subsequent regulatory event, perhaps phosphorylation, may be required for nuclear retention of TIM by PER.

In mammals, mPER2 stability is regulated by the MCRY proteins (Yagita et al., 2002), reminiscent of the manner in which TIM promotes PER stability in Drosophila. It was also shown recently that $\mathrm{mCRY}$ is not necessary for mPER2 nuclear entry but is necessary for its nuclear accumulation. Both proteins are actively exported via the same LMB-sensitive CRM1-exportin nuclear export pathways, and nuclear export is blocked when mCRY-mPER2 dimerization blocks their respective nuclear export NES signals (Yagita et al., 2002). As with many proteins, nuclear export was also linked to ubiquitylation and proteosomal degradation of mCRY and mPER2. Drosophila TIM and PER are also ubiquitinated and degraded by the proteasome, but the relevance of this for nuclear export is not known (Naidoo et al., 1999; Ko et al., 2002). Scanning TIM for the leucine-rich consensus sequence for CRM1-exportin-dependent nuclear export $\mathrm{Lx}_{(1-3)} \mathrm{Lx}_{(1-3)} \mathrm{LxL}(\mathrm{V} / \mathrm{I} / \mathrm{M})$ (in which $\mathrm{x}$ indicates any amino acid) (Yagita et al., 2002; Nigg, 1997 \#797) revealed six potential nuclear export signals (residues 184-193, 737-744, 750-758, 10461054, 1095-1102, and 1131-1139), none within the CLD region. We suspect that the modification of these sites by sgg phosphorylation will be the mechanism by which TIM becomes nuclear in the late evening. A similar scan of PER using this consensus sequence did not turn up any potential LMB-sensitive nuclear ex- port signals; however, Vielhabert et al. (2001) describe a conserved region in per that is an active NES in mPer 1 and mPer2.

This study challenges some of the assumptions of the current clock model concerning the effect of per dosage on circadian period and the regulation of PER-TIM nuclear entry and dimer function. It also indicates that TIM does not actively repress dCLK-CYC-mediated transcription and in fact may have a novel role in the nucleus. The active nuclear export of TIM provides yet another level of complexity to the function of TIM in the Drosophila circadian clock.

\section{References}

Bae K, Lee C, Sidote D, Chuang KY, Edery I (1998) Circadian regulation of a Drosophila homolog of the mammalian Clock gene: PER and TIM function as positive regulators. Mol Cell Biol 18:6142-6151.

Baylies MK, Bargielli TA, Jackson FR, Young MW (1987) Changes in abundance or structure of the per gene product can alter periodicity of the Drosophila clock. Nature 326:390-392.

Ceriani MF, Hogenesch JB, Yanovsky M, Panda S, Straume M, Kay SA (2002) Genome-wide expression analysis in Drosophila reveals genes controlling circadian behavior. J Neurosci 22:9305-9319.

Chen Y, Hunter-Ensor M, Schotland P, Sehgal A (1998) Alterations of per RNA in noncoding regions affect periodicity of circadian behavioral rhythms. J Biol Rhythms 13:364-379.

Claridge-Chang A, Wijnen H, Naef F, Boothroyd C, Rajewsky N, Young M (2001) Circadian regulation of gene expression systems in the Drosophila head. Neuron 32:657-671.

Curtin KD, Huang ZJ, Rosbash M (1995) Temporally regulated nuclear entry of the Drosophila period protein contributes to the circadian clock. Neuron 14:365-372.

Cyran SA, Buchsbaum AM, Reddy KL, Lin M, Glossop NRJ, Hardin PE, Young MW, Storti RV, Blau J (2003) vrille, Pdp1, and dClock form a second feedback loop in the Drosophila circadian clock. Cell 112:329-341.

Darlington TK, Wagner-Smith K, Ceriani MF, Staknis D, Gekakis N, Steeves TDL, Weitz CJ, Takahashi JS, Kay SA (1998) Closing the circadian loop: clock-induced transcription of its own inhibitors per and tim. Science 280:1599-1603.

Gekakis N, Saez L, Delahaye BA, Myers MP, Sehgal A, Young MW, Weitz CJ (1995) Isolation of timeless by PER protein interaction: defective interaction between timeless protein and long-period mutant PERL. Science 270:811-815.

Gill G, Pascal E, Tseng Z, Tjian R (1994) A glutamine-rich hydrophobic patch in transcription factor Sp1 contacts the dTAFII1 10 component of the Drosophila TFIID complex and mediates transcriptional activation. Proc Natl Acad Sci USA 91:192-196.

Glossop NRJ, Lyons LC, Hardin PE (1999) Interlocked feedback loops within the Drosophila circadian oscillator. Science 286:766-768.

Glossop N, Houl JH, Zheng H, Ng F, Dudek SM, Hardin P (2003) VRILLE feeds back to control circadian transcription of Clock in the Drosophila circadian oscillator. Neuron 37:249-261.

Hao H, Glossop N, Lyons L, Qiu J, Morrish B, Cheng Y, Helfrich-Forster C, Hardin P (1999) The 69 bp circadian regulatory sequence (CRS) mediates per-like developmental, spatial, and circadian expression and behavioral rescue in Drosophila. J Neurosci 19:987-994.

Helfrich-Forster C (1995) The period clock gene is expressed in central nervous system neurons which also produce a neuropeptide that reveals the projections of circadian pacemaker cells within the brain of Drosophila melanogaster. Proc Natl Acad Sci USA 92:612-616.

Henderson BR, Eleftheriou A (2000) A comparison of the activity, sequence specificity, and CRM1-dependence of different nuclear export signals. Exp Cell Res 256:213-224.

Hirayama J, Fukuda I, Ishikawa T, Kobayashi K, Todo T (2003) New role of zCRY and zPER2 as regulators of subcellular distributions of zCLOCK and zBMAL proteins. Nucleic Acids Res 31:935-943.

Hunter-Ensor M, Ousley A, Sehgal A (1996) Regulation of the Drosophila protein timeless suggests a mechanism for resetting the circadian clock by light. Cell 84:677-686.

Ivachenko M, Stanewsky R, Giebultowicz JM (2001) Circadian photoreception in Drosophila: functions of cryptochrome in peripheral and central clocks. J Biol Rhythms 16:205-215.

Kaneko M, Helfrich-Forster C, Hall JC (1997) Spatial and temporal expres- 
sion of the period and timeless genes in the developing nervous system of Drosophila: newly identified pacemaker candidates and novel features of clock gene product cycling. J Neurosci 17:6745-6760.

Ko HW, Jiang J, Edery I (2002) Role for Slimb in the degradation of Drosophila Period protein phosphorylated by Doubletime. Nature 420:673-678.

Kobayashi Y, Ishikawa T, Hirayama J, Daiyasu H, Kanai S, Toh H, Fukuda I, Twujimura T, Terada N, Kamei Y, Yuba S, Iwai S, Todo T (2000) Molecular analysis of zebrafish photolyase/cryptochrome family: two types of cryptochromes present in zebrafish. Genes Cells 5:725-738.

Krishnan B, Levine JD, Lynch MKS, Dowse HB, Funes P, Hall JC, Hardin PE, Dryer SE (2001) A new role for cryptochrome in a Drosophila circadian oscillator. Nature 411:313-317.

Kume K, Zylka MJ, Sriram S, Shearman LP, Weaver DR, Jin X, Maywood ES, Hastings MH, Reppert SM (1999) mCRY1 and mCRY2 are essential components of the negative limb of the circadian clock feedback loop. Cell 98:193-205.

Lee C, Parikh V, Itsukaichi T, Bae K, Edery I (1996) Resetting the Drosophila clock by photic regulation of PER and a PER-TIM complex. Science 271:1740-1744.

Lee C, Bae K, Edery I (1998) The Drosophila CLOCK protein undergoes daily rhythms in abundance, phosphorylation and interactions with the PER-TIM complex. Neuron 21:857-867.

Lee C, Bae K, Edery I (1999) PER and TIM inhibit the DNA binding activity of a Drosophila CLOCK-CYC/dBMAL1 heterodimer without disrupting formation of the heterodimer: a basis for circadian transcription. Mol Cell Biol 19:5316-5325.

Lin F, Song W, Meyer-Bernstein E, Naidoo N, Sehgal A (2001) Photic signaling by cryptochrome in the Drosophila circadian clock. Mol Cell Biol 21:7287-7294.

Lin Y, Han M, Shimada B, Wang L, Gibler TM, Amarakone A, Awad TA, Stomo GD, Van Gelder RN, Taghert PH (2002) Influence of the perioddependent circadian clock on diurnal, circadian, and aperiodic gene expression in Drosophila melanogaster. Proc Natl Acad Sci USA 99:9562-9567.

Martinek S, Inonog S, Manoukian AS, Young MW (2001) A role for the segment polarity gene shaggy/GSK-3 in the Drosophila circadian clock. Cell 105:769-779.

McDonald MJ, Rosbash M (2001) Microarray analysis and organization of circadian gene expression in Drosophila. Cell 107:567-578.

Myers MP, Wager-Smith K, Rothenflugh A, Young MW (1996) Lightinduced degradation of TIMELESS and entrainment of the Drosophila circadian clock. Science 271:1736-1740.

Naidoo N, Song W, Hunter-Ensor M, Sehgal A (1999) A role for the proteasome in the light response of the timeless clock protein. Science 285:1737-1741.

Nishi K, Yoshida M, Fujiwara D, Nishikawa M, Horinouchi S, Beppu T (1994) Leptomycin B targets a regulatory cascade of crml, a fission yeast nuclear protein involved in control of higher order chromosome structure and gene expression. J Biol Chem 269:6320-6324.

Ousley A, Zafarullah K, Chen Y, Emerson M, Hickman L, Sehgal A (1998) Conserved regions of the timeless ( $\mathrm{tim}$ ) clock gene in Drosophila analyzed through phylogenetic and functional studies. Genetics 148:815-825.
Price JL, Dembinska ME, Young MW, Rosbash M (1995) Suppression of PERIOD protein abundance and circadian cycling by the Drosophila clock mutation timeless. EMBO J 14:4044-4049.

Roberts S (2000) Mechanisms of action of transcription activation and repression domains. Cell Mol Life Sci 57:1149-1160.

Rosato E, Veryan C, Mazzotta G, Piccin A, Zordan M, Costa R, Kyriacou C (2001) Light-dependent interaction between Drosophila CRY and the clock protein PER mediated by the carboxy terminus of CRY. Curr Biol 11:908-917.

Rothenfluh A, Young MW, Saez L (2000a) A TIMELESS-independent function for PERIOD proteins in the Drosophila clock. Neuron 26:505-514.

Rothenfluh A, Abodeely M, Price JL, Young MW (2000b) Isolation and analysis of six timeless alleles that cause short or long period circadian rhythms in Drosophila. Genetics 156:665-675.

Saez L, Young MW (1996) Regulation of nuclear entry of the Drosophila clock proteins Period and Timeless. Neuron 17:911-920.

Schotland P, Hunter-Ensor M, Lawrence T, Sehgal A (2000) Altered entrainment and feedback loop function effected by a mutant period protein. J Neurosci 20:958-968.

Shafer O, Rosbash M, Truman J (2002) Sequential nuclear accumulation of the clock proteins period and timeless in the pacemaker neurons of Drosophila melanogaster. J Neurosci 22:5946-5954.

Smith R, Konopka R (1982) Effects of dosage alterations at the per locus on the period of the circadian clock of Drosophila. Mol Gen Genet 185:30-36

So WV, Rosbash M (1997) Post-transcriptional regulation contributes to Drosophila clock gene mRNA cycling. EMBO J 16:7146-7155.

Stanewsky R (2003) Genetic analysis of the circadian system in Drosophila melanogaster and mammals. J Neurobiol 54:111-147.

Suri V, Lanjuin A, Rosbash M (1999) TIMELESS-dependent positive and negative autoregulation in the Drosophila circadian clock. EMBO J 18:675-686.

Thummel CS, Pirrotta V (1992) New pCaSpeR element vectors. DIS 71:150.

Vielhabert EL, Duricka D, Ullman KS, Virshup D (2001) Nuclear export of mammalian PERIOD proteins. J Biol Chem 276:45921-45927.

Vosshall LB, Price JL, Sehgal A, Saez L, Young MW (1994) Block in nuclear localization of period protein by a second clock mutation, timeless. Science 263:1606-1609.

Wang G, Ousley A, Darlington T, Chen D, Chen Y, Fu W, Hickman L, Kay S, Sehgal A (2001) Regulation of the cycling of timeless RNA. J Neurobiol 47:161-175.

Williams JA, Sehgal A (2001) Molecular components of the Drosophila circadian clock. Annu Rev Physiol 63:729-755.

Yagita K, Tamanini F, Yasuda M, Hoeijmakers JHJ, Van der Horst GTJ, Okamura H (2002) Nucleocytoplasmic shuttling and mCRY-dependent inhibition of ubiquitylation of the mPER2 clock protein. EMBO J 21:1301-1314.

Yang Z, Sehgal A (2001) Role of molecular oscillations in the Drosophila circadian clock. Neuron 29:453-467.

Zeng H, Qian Z, Myers MP, Rosbash M (1996) A light entrainment mechanism for the Drosophila circadian clock. Nature 380:129-135. 